\title{
Enrichment Strategies in Pediatric Drug Development: An Analysis of Trials Submitted to the US Food and Drug Administration
}

Dionna J. Green ${ }^{1}$, Xiaomei I. Liu' ${ }^{1}$, Tianyi Hua ${ }^{2}$, Janelle M. Burnham ${ }^{1}$, Robert Schuck ${ }^{1}$, Michael Pacanowski ${ }^{1}$, Lynne Yao ${ }^{3}$, Susan K. McCune ${ }^{4}$, Gilbert J. Burckart ${ }^{1}$, Issam Zineh ${ }^{1}$

${ }^{1}$ Office of Clinical Pharmacology, Center for Drug Evaluation and Research, US Food and Drug Administration, Silver Spring, MD; ${ }^{2}$ University of Michigan College of Pharmacy, Ann Arbor, MI; ${ }^{3}$ Office of New Drugs, Center for Drug Evaluation and Research, US Food and Drug Administration, Silver, Spring, MD; ${ }^{4}$ Office of Pediatric Therapeutics, Commissioner's Office, US Food and Drug Administration, Silver Spring, MD.

Disclaimer: The opinions expressed in this article are those of the authors and should not be interpreted as the position of the U.S. Food and Drug Administration.

Funding: No funding was received for this work.

Conflict of Interest: The authors declared no competing interests for this work.

Corresponding author: Dionna Green, MD, Medical Officer / Policy Lead, Office of Clinical Pharmacology, Center for Drug Evaluation and Research, US Food and Drug Administration, 10903 New Hampshire Ave, Silver Spring, MD 20903

Tel: (301) 796-1543, FAX 301-847-8720; E-mail: Dionna.Green@fda.hhs.gov

Keywords: enrichment, pediatric, drug development, US FDA

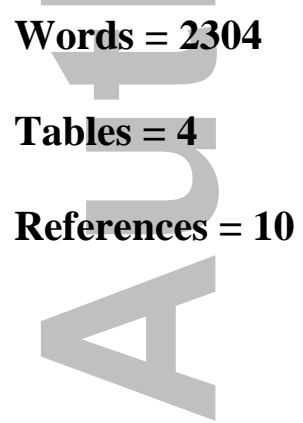

This is the author manuscript accepted for publication and has undergone full peer review but has not been through the copyediting, typesetting, pagination and proofreading process, which may lead to differences between this version and the Version of record. Please cite this article as doi:10.1002/ cpt.971. 


\section{Abstract}

Clinical trial enrichment involves prospectively incorporating trial design elements that increase the probability of detecting a treatment effect. The use of enrichment strategies in pediatric drug development has not been systematically assessed. We analyzed the use of enrichment strategies in pediatric trials submitted to the US Food and Drug Administration from 2012-2016. One hundred twelve efficacy studies associated with 76 drug development programs were assessed and their overall success rates were $78 \%$ and $75 \%$ respectively. Eighty-eight trials (76.8\%) employed at least one enrichment strategy; of these, $66.3 \%$ employed multiple enrichment strategies. The highest trial success rates were achieved when all three enrichment strategies (practical, predictive, and prognostic) were used together within a single trial (87.5\%), while the lowest success rate was observed when no enrichment strategy was used (65.4\%). The use of enrichment strategies in pediatric trials was found to be associated with trial and program success in our analysis.

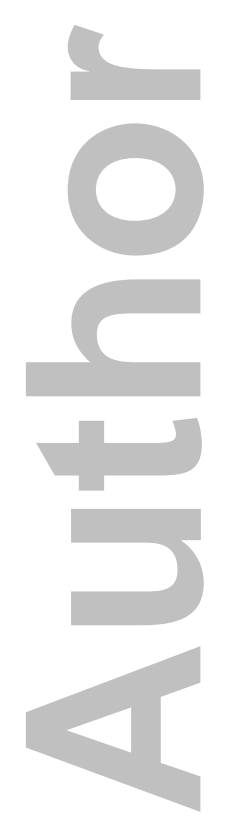




\section{Introduction}

Designing clinical trials to include patient populations most likely to demonstrate a response to the therapy being studied is not a new concept. The term "enrichment" refers to the prospective incorporation of clinical trial design elements intended to maximize the likelihood of observing a drug's treatment effect (if, in fact, the drug is effective), and has been a focus area of the US Food and Drug Administration (FDA) since 1994.(1, 2) Enrichment strategies have been promulgated by FDA over the ensuing decades, culminating in the 2012 FDA Guidance for Industry: Enrichment Strategies for Clinical Trials to Support Approval of Human Drugs and Biological Products.(3) A variety of enrichment strategies exist, including those intended to 1) reduce non-drug-related variability (practical enrichment);2) increase the incidence of clinical events of interest (prognostic enrichment); or 3) select patients most likely to respond to treatment (predictive enrichment). All of these maneuvers are intended to increase study power to detect an investigational drug's treatment effect. Enrichment may, therefore, be particularly valuable when there are a limited number of patients available for study, or when there have been difficulties in achieving successful clinical trials for a therapeutic indication. Both situations fit pediatric drug development today.

Pediatric drug development is a relatively new science. The Best Pharmaceuticals for Children Act (BPCA) in 2002 and the Pediatric Research Equity Act (PREA) in 2003 were essentially the starting point for what has become a significant effort by drug developers and regulatory agencies to test new products in pediatric patients. The FDA has now received results from well over 1,000 pediatric clinical trials. Many of these trials fail to show treatment benefit in the pediatric population, resulting in inability to approve the tested drugs for pediatric use. In one systematic review of studies submitted to the FDA from 1998 - 2012 for which sponsors 
were seeking pediatric exclusivity, $42 \%$ of pediatric drug development studies failed(4), and there are multiple reasons as to why these trials failed.(5) Optimizing enrichment strategies for pediatric trials appears to be a reasonable approach to increase trial success rates. In fact, in some pediatric diseases, enrichment has been one method of turning an area with failed trials into an area of successful pediatric drug development.(6, 7) A systematic evaluation of enrichment strategy approaches in pediatric drug development trials has not been performed to date. The objectives of this analysis are to describe the use of enrichment strategies in pediatric drug development trials submitted to the FDA from 2012-2016 and assess the impact of enrichment on trial outcome and pediatric drug approval.

\section{Results}

Under FDASIA, 116 pediatric efficacy studies were identified, and 112 studies had all materials, including the pediatric protocols, available at the time of review. The 112 studies were associated with 76 pediatric drug development programs, with some programs involving more than one pediatric trial (range 1-4). The therapeutic areas and related approval rates for both trials and programs are summarized in Table 2. There was a range of success rates at both the clinical trial and drug development program levels. The overall success rate across therapeutic areas at the trial and program levels were $78 \%$ (median $81.8 \%$ and interquartile range [IQR] 53.4\%) and 75\% (median 80.8\% and IQR 35.4\%), respectively. Of the 76 programs, 42 $(55.3 \%)$ used at least one prognostic strategy, 30 (39.5\%) used at least one practical strategy, and $27(35.5 \%)$ used at least one predictive strategy. Table 3 lists the therapeutic areas, enrichment strategies used, and trial outcome. Overall, prognostic strategies constituted the most frequently used strategy (41.5\%), followed by practical (30.8\%) and predictive (27.7\%) strategies. 
Table 4 lists the composite use of strategies in trials and their success rates. Of the 112 clinical trials evaluated, a large majority (76.8\%) employed at least one enrichment strategy. Of the studies that employed enrichment strategies $(n=86), 66.3 \%$ employed multiple enrichment strategies, while $33.7 \%$ used a single-enrichment approach. In clinical trials where only one, enrichment strategy was used, the most common strategy was prognostic enrichment (44.8\%), followed by predictive enrichment (37.9\%), and practical enrichment (17.3\%). In trials that used multiple enrichment strategies, the highest success rates were achieved when all three enrichment strategies were used together $(87.5 \%)$ compared with when two strategies were used (clinical trial success rate range $75 \%-79.2 \%$ ). Of particular note, the lowest clinical trial success rate was observed when no enrichment strategy was used (65.4\% vs. $81.4 \%$ for use of any enrichment strategy).

\section{Discussion}

The need for pediatric patient access to safe and effective FDA-approved medications has been long recognized and served as a major impetus for BPCA and PREA. Since the passage of these laws, there has been increased attention to 1) root causes of failure in pediatric drug development; and 2) approaches to increase the probability of pediatric clinical trial and drug development program success. Clinical trial enrichment represents a powerful strategy for selecting a subset of the general population in which the effect of the drug can be more efficiently demonstrated. This approach has the potential to result in smaller studies, increased study power, and/or shortened drug development times. As such, enrichment is particularly attractive for pediatric drug development studies which are often plagued with small disease populations, enrollment challenges, and a relatively high failure rate of establishing efficacy. 
Given that there is often only one opportunity to evaluate a given drug in a pediatric drug development study, utilizing trial design tactics, such as enrichment, that offer the highest prospect of trial success is critical. To our knowledge this is the first study to systematically evaluate the use of enrichment strategies in pediatric clinical trials. We observed several important findings: (1) enrichment is a common feature in pediatric trials, with the vast majority of trials included in our cohort incorporating at least one enrichment strategy for patient selection; (2) in general, prognostic enrichment strategies were the most commonly used; (3) the highest trial success rates were observed when all three enrichment approaches (practical, predictive, prognostic) were used in a single trial; and (4) the rate of trial success was higher for those trials that employed an enrichment design compared to trials that did not contain any enrichment maneuvers.

The high frequency of use of enrichment strategies in pediatric drug development that we observed could be the result of several factors. In general, pediatric drug development temporally follows adult drug development. During adult drug development, significant knowledge is gained about variability in the investigational drug response, variability in placebo (or control) response, performance of the primary and secondary clinical trial endpoints, effect sizes, tolerability, and dose- or exposure-response relationships. Furthermore, more mechanistic or disease understanding can emerge during the lag between adult and pediatric development programs. As this knowledge accumulates, more informed clinical trials can be designed and conducted in subsequent populations of interest, including in pediatric populations.

In our analysis, prognostic enrichment was generally the most common type of enrichment (after accounting for universally employed practical enrichment strategies as described in Methods above). Specifically, over 55\% of the 76 programs evaluated used at least 
one prognostic enrichment strategy, compared with the approximately $36 \%$ and $40 \%$ use of predictive and practical enrichment, respectively. Additionally, prognostic enrichment was overrepresented $(\sim 45 \%)$ in clinical trials that employed a single enrichment strategy compared with those that used predictive $(\sim 38 \%)$ or practical $(\sim 17 \%)$ enrichment as their single enrichment approach. The more frequent use of prognostic enrichment may reflect greater knowledge of and confidence around the risk factors associated with the clinical events of interest in the diseases studied compared with our generally limited understanding of clearly identifiable predictors of drug response.

Interestingly, the majority of development programs not only used enrichment approaches, but often used multiple enrichment approaches simultaneously. When looking at trials that used more than one enrichment approach, trials that employed all three strategies (i.e., practical, prognostic, and predictive) demonstrated nominally greater success rate (approaching 90\%) compared with those that employed any two strategies in combination (success rates ranged from $75-79 \%$ ). The ability to use all three enrichment approaches may be indicative of a more holistic understanding of 1) the determinants of drug response variability, 2) disease mechanism and manifestation in pediatric populations, and 3) other sources of non-drug related variability. A thorough assessment of these factors, if known, prior to the conduct of clinical trials in pediatric populations is prudent, and should be documented and disseminated when possible to enable pediatric drug development across a wide range of therapeutic areas.

Of critical importance, our data suggest that use of any type of enrichment approach is more likely to lead to clinical trial and development program success compared to not using enrichment as part of clinical trial design and execution. Clinical trials that used enrichment were 1.24 times more likely to successfully meet their pre-specified primary endpoint compared 
with trials that used no enrichment strategy. In total, our data suggest the ability to enrich a clinical trial does seem to be an important determinant of clinical trial success in pediatric drug development.

Prior to our analysis, there had been anecdotal evidence that enrichment strategies in specific pediatric patient populations could lead to drug development success and approval of treatment in diseases that had historically shown failure. For example, in the case of development of drugs to treat migraine headaches in adolescents, early clinical trials all failed between 1999 and 2003. Root cause analyses revealed that the high placebo response rate in this population was a major driver of clinical trial failure. Consequently, more recent trials employed an enrichment approach that double-randomized patients and dropped placebo responders after the first randomization, leading to successful trials of drugs in adolescent migraine headache.(7) Another example of the use of enrichment strategies in specific patient populations is the study of oxybutynin for pediatric neurogenic bladder dysfunction. A predictive enrichment strategy was employed in this trial which involved the inclusion of pediatric patients who were current users of oxybutynin and had previously responded to and tolerated the drug, and therefore were more likely to respond to treatment. Patients who experienced adverse effects or did not respond to oxybutynin were excluded from the trial.(6) Our systematic review lends support to these anecdotal cases in which enrichment strategies increased the likelihood for pediatric clinical trial success.

(1)

Our current analysis is not without limitations. First, our cohort is comprehensive for the time period evaluated (2012-2016). Notwithstanding, the cohort consisted of studies submitted to the FDA during this time period, not necessarily conducted during this time period. In fact, the studies were in large part designed and executed prior to 2012. Consequently, we were not 
able to assess the impact of the 2012 FDA enrichment guidance on the prevalence of enrichment strategies in pediatric trials. Second, while comprehensive for the time period evaluated, the relatively small number of trials within some of the therapeutic areas listed limits the ability to make definitive conclusions about the utility of enrichment strategies in specific therapeutic areas. Third, a number of other factors are known to influence clinical trial outcome; these include, but are not limited to, appropriateness of the dosing regimen tested and definition and ascertainment of the primary endpoint studied. A multidimensional evaluation of all factors that could be associated with clinical trial success or failure was beyond the scope of our present work, and may not ultimately be feasible given the limited sample of trials and development programs. Nonetheless, the totality of the data suggests the importance of enrichment as a consideration in pediatric clinical trial planning.

In conclusion, our review of 112 pediatric drug development trials in 76 drug development program from 2012-2016 identified extensive use of practical, prognostic, and predictive enrichment strategies in pediatric drug development. The combined use of multiple enrichment strategies was common, but some programs used no enrichment strategies. In general, the use of enrichment strategies was associated with successful pediatric trials and programs. The use of enrichment in pediatric drug development programs, where patients are often limited and ethical concerns also restrict enrollment of pediatric patients, should continue to be encouraged. To the extent possible, enrichment designs should be explicitly considered in protocol development and clinical trial execution in pediatric drug development.

\section{Methods}

We surveyed pediatric efficacy trials from drug development programs submitted to FDA under the Food and Drug Administration Safety and Innovation Act (FDASIA, July, 2012 - July, 
2016). Only trials that were deemed as pivotal in supporting a labeling claim for efficacy in the pediatric population were reviewed as part of this survey. For each trial and drug product, the pediatric protocols, publically available FDA reviews, $(8,9)$ and the product labels $(10)$ were reviewed.

FDA-authored reviews of each trial and the trial protocols were used to extract the following data: drug name, proposed indication (i.e., pediatric condition for which the approval was being sought), FDA therapeutic review area, enrichment strategy(ies) used, trial outcome and drug approval status.

Enrichment strategies were categorized broadly as practical, predictive, or prognostic. Strategies were subsequently further subcategorized as outlined in the 2012 FDA draft Guidance for Industry: Enrichment Strategies for Clinical Trials to Support Approval of Human Drugs and Biological Products (Table 1).(3) The classification of enrichment strategies used in pediatric drug development programs was adjudicated by three authors and disagreements were resolved by consensus. Universally employed practical enrichment strategies, such as the exclusion of patients with hypersensitivities to the drug under study, with concomitant illnesses likely to lead to drop-out or death, or who are taking drugs that pharmacologically similar to or likely to interact with the study drug, were not included in the analysis.

Trial outcome was categorized as a success or failure based upon whether or not the trial achieved statistical significance on its primary efficacy endpoint. Drug approval status was categorized as approved or not approved based upon whether the pivotal trial(s) resulted in an FDA-approved indication for use of the drug in the pediatric population studied. 


\section{Study Highlights (150 words - excluding question as per author guide)}

\section{WHAT IS THE CURRENT KNOWLEDGE ON THE TOPIC?}

○ Clinical trial enrichment involves prospectively incorporating trial design elements that increase the probability of detecting a treatment effect, and an FDA guidance for enrichment is available.

\section{- WHAT QUESTION DOES THIS STUDY ADDRESS?}

Pediatric drug development is a new science, and the use of enrichment strategies in pediatric drug development has not been systematically assessed.

\section{WHAT DOES THIS STUDY ADD TO OUR KNOWLEDGE?}

(
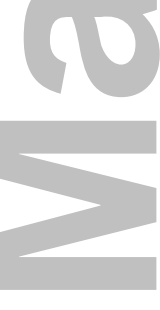

\section{HOW MIGHT THIS CHANGE CLINICAL PHARMACOLOGY OR}

\section{TRANSLATIONAL SCIENCE?}

The use of practical, prognostic and predictive enrichment strategies in designing pediatric drug development studies is a critical part of increasing clinical trial success, and should be formulated with the same rigor as other parts of pediatric trial design. 
Disclaimer: The opinions expressed in this article are those of the authors and should not be interpreted as the position of the U.S. Food and Drug Administration.

Acknowledgments: The authors would like to acknowledge the contributions of pharmacy students Joseph Chen, Michael Hwang and Karey Kowalski to this work. The results from this study were presented in part at an FDA public workshop (September 2017) and at the American College of Clinical Pharmacology Annual Meeting in (September 2017).

Disclosure: The authors declare no potential conflicts of interest with respect to the research, authorship, and/or publication of this article. The authors received no financial support for the research, authorship, and/or publication of this article.

\section{Author Contributions}

D.J.G., J.M.B., G.J.B., R.S., M.P., L.Y., S.K.McC., and I.Z. wrote the manuscript; D.J.G., J.M.B., G.J.B., R.S., M.P., and I.Z. designed the research; D.J.G., X.I.L., and T.H. performed the research; D.J.G., X.I.L., J.M.B., T.H., G.J.B., and I.Z. analyzed the data.

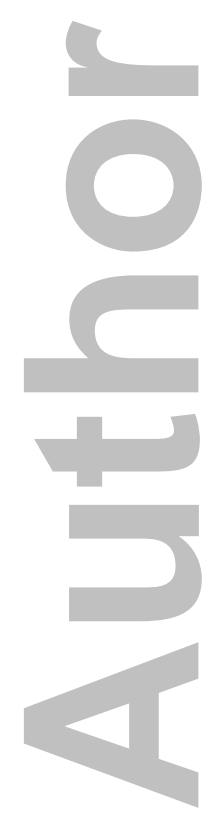




\section{References}

1. Temple R. Early Escape, Enrichment, Studies in Non-Responders. Commun Stat Theory Meth. 1994;23:499-531.

2. Temple R. Enrichment of clinical study populations. Clin Pharmacol Ther. 2010;88(6):774-8.

3. Guidance for Industry: Enrichment Strategies for Clinical Trials to Support Approval of Human Drugs and Biological Products. US Food and Drug Administration; 2012.

4. Wharton G.T. et al. Impact of pediatric exclusivity on drug labeling and demonstrations of efficacy. Pediatrics. 2014;134(2):e512-8.

5. Momper J.D., Mulugeta Y., Burckart G.J. Failed Pediatric Drug Development Trials. Clin Pharmacol Ther. 2015;98(3):245-51.

6. Momper J.D. et al. Drug development for pediatric neurogenic bladder dysfunction: dosing, endpoints, and study design. J Clin Pharmacol. 2014;54(11):1239-46.

7. Sun H. et al. Migraine therapeutics in adolescents: a systematic analysis and historic perspectives of triptan trials in adolescents. JAMA Pediatr. 2013;167(3):243-9.

8. Reviews of Pediatric Studies Conducted under BPCA and PREA from 2007-2012. Available from:

https://www.fda.gov/Drugs/DevelopmentApprovalProcess/DevelopmentResources/ucm049872.h $\underline{\operatorname{tm}}$.

9. Reviews of Pediatric Studies Conducted under BPCA and PREA from 2012 - present. Available from: https://www.fda.gov/Drugs/DevelopmentApprovalProcess/DevelopmentResources/ucm316937.h $\underline{\operatorname{tm}}$. 
10. Drugs@FDA: FDA Approved Drug Products. Available from:

https://www.accessdata.fda.gov/scripts/cder/daf/index.cfm.

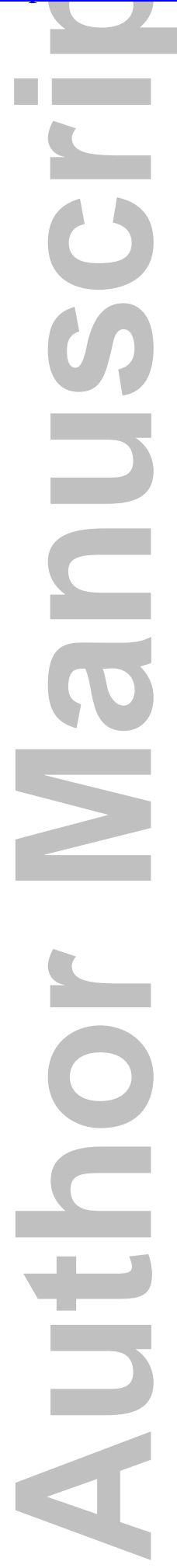

This article is protected by copyright. All rights reserved. 
Table 1. Enrichment strategies *
Strategy
Practical

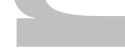
4
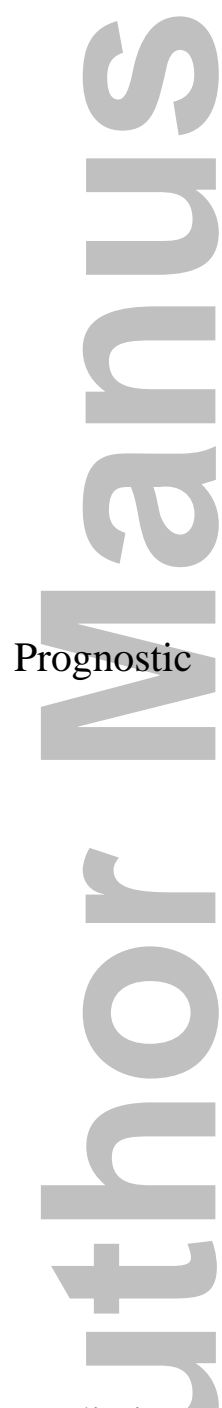
Predictive

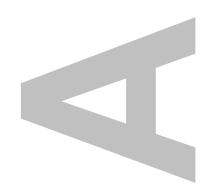
Enrichment Approach Description
Pre-randomization compliance Prior to randomization, identifying and selecting patients likely to comply with treatment
Placebo run-in (placebo
Using a lead-in period prior to responder) randomization to eliminate patients who improve spontaneously or have large placebo responses
Including only patients with consistent baseline values
Medical history
Enrolling only patients who have consistent baseline values (e.g. blood pressure measurements, pulmonary function tests)
Selecting patients with a greater likelihood of having an event (endpoint) based on their medical history
Clinical / laboratory measurement
Selecting patients with a greater likelihood of having an event based on a clinical or laboratory measurement
Genomic / proteomic
Selecting patients with a measurement greater likelihood of having an event based on genomic or proteomic measures
Active run-in or history of response to the medication
Selecting patients with a past history of response to the particular intervention under study either based on past history of use or using an active run-in period during the 


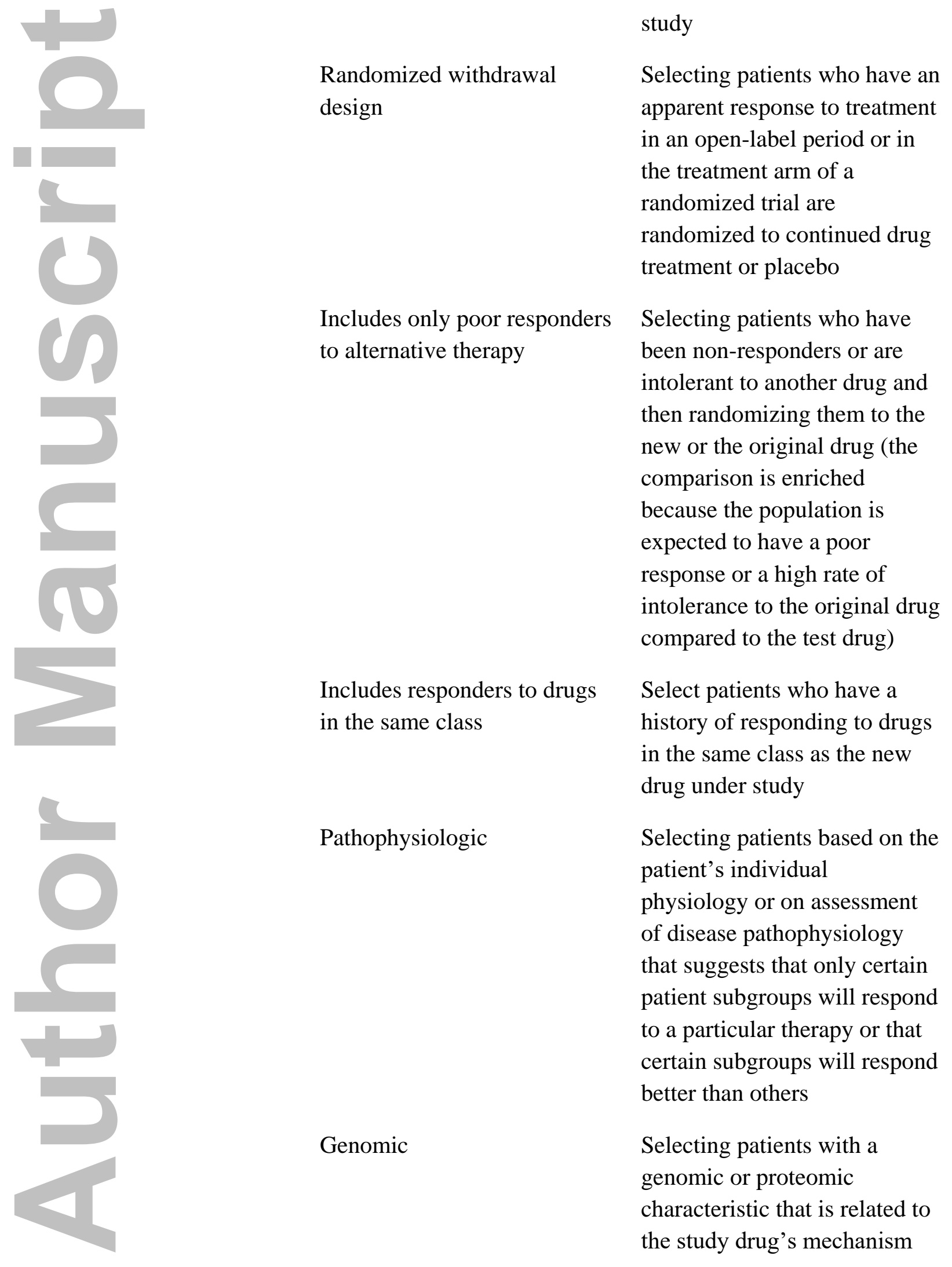




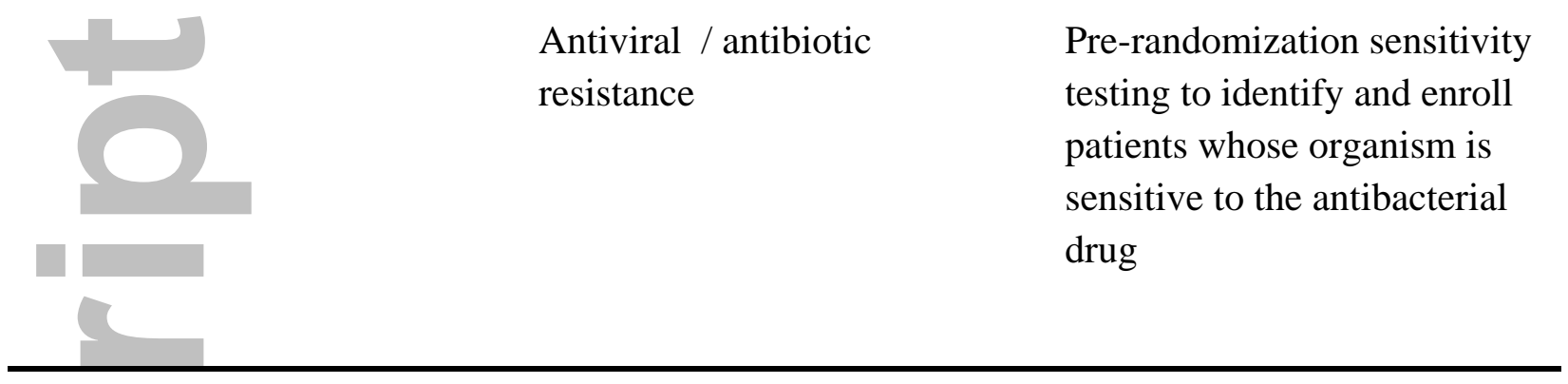

*Guidance for Industry: Enrichment Strategies for Clinical Trials to Support Approval of Human Drugs and Biological Products. US Food and Drug Administration; 2012.

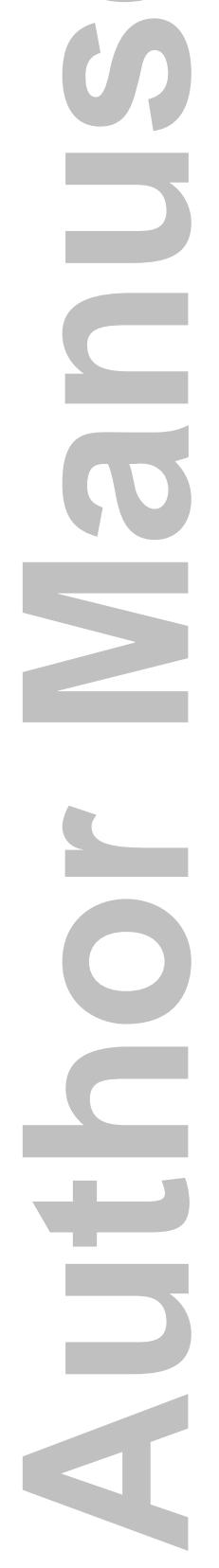


Table 2. Therapeutic area, trial outcome, and program outcome.

\begin{tabular}{|c|c|c|c|c|}
\hline $\begin{array}{l}\text { Therapeutic } \\
\text { Area }\end{array}$ & $\begin{array}{l}\text { Trial, } \\
\mathrm{N}\end{array}$ & $\begin{array}{l}\text { Trial } \\
\text { success, N (\%) }\end{array}$ & $\begin{array}{l}\text { Program, } \\
\mathrm{N}\end{array}$ & $\begin{array}{l}\text { Program } \\
\text { success }^{\dagger}, \mathrm{N}(\%)\end{array}$ \\
\hline Anti-infectives & 12 & $12(100.0 \%)$ & 6 & $6(100.0 \%)$ \\
\hline Antiviral & 15 & $15(100.0 \%)$ & 11 & $11(100.0 \%)$ \\
\hline Dermatology & 4 & $4(100.0 \%)$ & 3 & $3(100.0 \%)$ \\
\hline Hematology & 4 & $4(100.0 \%)$ & 3 & $3(100.0 \%)$ \\
\hline Imaging & 1 & $1(100.0 \%)$ & 1 & $1(100.0 \%)$ \\
\hline Inborn error & 1 & $1(100.0 \%)$ & 1 & $1(100.0 \%)$ \\
\hline Neurology & 11 & $9(81.8 \%)$ & 6 & $5(83.3 \%)$ \\
\hline Allergy & 8 & $6(75.0 \%)$ & 5 & $4(80.0 \%)$ \\
\hline GI & 5 & $3(60.0 \%)$ & 5 & $4(80.0 \%)$ \\
\hline \multicolumn{5}{|l|}{ Analgesia/ } \\
\hline Anesthesia & 6 & $2(33.3 \%)$ & 3 & $2(66.7 \%)$ \\
\hline Pulmonary & 19 & $17(89.5 \%)$ & 9 & $6(66.7 \%)$ \\
\hline Psychiatry & 19 & $12(63.2 \%)$ & 16 & $10(62.5 \%)$ \\
\hline Oncology & 4 & $1(25.0 \%)$ & 4 & $1(25.0 \%)$ \\
\hline Cardio-renal & 1 & $0(0.0 \%)$ & 1 & $0(0.0 \%)$ \\
\hline Ophthalmology & 2 & $0(0.0 \%)$ & 2 & $0(0.0 \%)$ \\
\hline TOTAL & 112 & $87(77.7 \%)$ & 76 & $57(75.0 \%)$ \\
\hline
\end{tabular}

*Rank ordered by program success

'Program success was defined as a program that resulted in an FDA-approved indication for use of the drug in the pediatric population studied 
Table 3. Therapeutic area, enrichment strategies employed, and trial outcomes.

\begin{tabular}{|c|c|c|c|c|}
\hline $\begin{array}{l}\text { Therapeutic } \\
\text { Area }\left(\mathrm{N}^{*}\right)\end{array}$ & $\begin{array}{l}\text { Practical } \\
\text { Strategy, } N^{\dagger}\end{array}$ & $\begin{array}{l}\text { Prognostic } \\
\text { Strategy, } \mathrm{N}^{\dagger}\end{array}$ & $\begin{array}{l}\text { Predictive } \\
\text { Strategy, } N^{\dagger}\end{array}$ & $\begin{array}{l}\text { Trial succes: } \\
\mathrm{N}^{*}(\%)\end{array}$ \\
\hline Anti-infectives (12) & 0 & 4 & 4 & $12(100.0 \%)$ \\
\hline Antiviral (15) & 1 & 1 & 6 & $15(100.0 \%)$ \\
\hline Dermatology (4) & 0 & 2 & 0 & $4(100.0 \%)$ \\
\hline Hematology (4) & 2 & 4 & 2 & $4(100.0 \%)$ \\
\hline Imaging (1) & 0 & 0 & 0 & $1(100.0 \%)$ \\
\hline Inborn error (1) & 1 & 0 & 1 & $1(100.0 \%)$ \\
\hline Pulmonary (19) & 17 & 18 & 12 & $17(89.5 \%)$ \\
\hline Neurology (11) & 10 & 11 & 1 & $9(81.8 \%)$ \\
\hline Allergy (8) & 8 & 8 & 2 & $6(75.0 \%)$ \\
\hline Psychiatry (19) & 10 & 12 & 13 & $12(63.2 \%)$ \\
\hline GI (5) & 0 & 2 & 0 & $3(60.0 \%)$ \\
\hline \multicolumn{5}{|l|}{ Analgesia/ } \\
\hline Anesthesia (6) & 0 & 3 & 2 & $2(33.3 \%)$ \\
\hline Oncology (4) & 0 & 1 & 1 & $1(25.0 \%)$ \\
\hline Cardio-renal (1) & 0 & 0 & 0 & $0(0.0 \%)$ \\
\hline Ophthalmology (2) & 0 & 0 & 0 & $0(0.0 \%)$ \\
\hline TOTAL (112) & 49 & 66 & 44 & $87(77.7 \%)$ \\
\hline \multicolumn{5}{|l|}{ * Number of trials } \\
\hline${ }^{\dagger}$ Number of strategie & & & & \\
\hline
\end{tabular}


Table 4. Overall trial success rates by enrichment strategies employed.

Enrichment strategy

Total trials Trial success Success rate $\%$

Practical + Prognostic + Predictive

16

14

87.5

Practical + Prognostic

24

19

79.2

Practical + Predictive

4

3

75.0

Prognostic + Predictive

13

10

76.9

Practical only

5

4

80.0

Prognostic only

13

10

76.9

Predictive only

11

10

90.9

Any strategy

86

70

81.4

No enrichment strategy

26

17

65.4

TOTAL

112

87

77.7

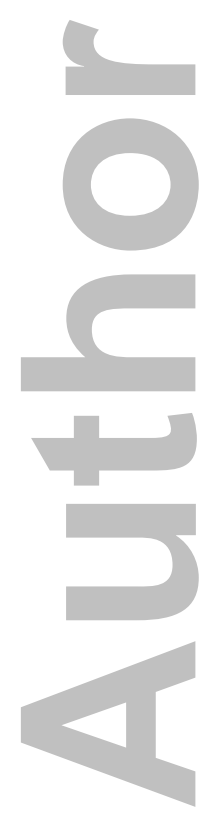

